\title{
ON THE NORMAL BUNDLE OF A SPHERE IMBEDDED IN EUCLIDEAN SPACE ${ }^{1}$
}

\author{
W. S. MASSEY
}

1. Introduction. An important question in the topology of differentiable manifolds is the following: Is the normal bundle of a compact, orientable differentiable manifold imbedded ${ }^{2}$ in Euclidean space intrinsically determined by the manifold, independent of the imbedding? This question may be rephrased more precisely as follows: For any two imbeddings of a compact, orientable, differentiable $n$-manifold in $(n+k)$-dimensional Euclidean space, are the normal bundles equivalent? Apparently this question is rather difficult, and very little is known about it. The following are the principal known facts:

(a) The characteristic classes of the normal bundle are intrinsically determined, independent of the imbedding. (The Stiefel-Whitney classes and the Pontrjagin classes are determined by the duality theorems. The Euler-Poincaré class of the normal bundle is always 0 .)

(b) The normal bundle to a compact, orientable $n$-manifold imbedded in $(n+1)$ or $(n+2)$-dimensional Euclidean space is always trivial. This is well known, but we sketch a proof in $\$ 2$ for the sake of completeness.

(c) The normal bundle to an $n$-sphere imbedded in $(n+k)$-dimensional Euclidean space is trivial if $k>(n+1) / 2$. This is a recent result $^{3}$ of M. Kervaire [4].

(d) If an $n$-manifold can be imbedded in some Euclidean space with a trivial normal bundle, then every imbedding in $(n+k)$ dimensional Euclidean space with $k \geqq n$ has a trivial normal bundle. This is an unpublished result of M. Kervaire.

Probably the simplest case to consider is that of an $n$-sphere, or a manifold which is like an $n$-sphere, imbedded in Euclidean space. The purpose of this paper is to prove the following theorem for this case:

Received by the editors March 11, 1959.

1 During the preparation of this paper, the author was partially supported by a grant from the National Science Foundation. An abstract announcing the results of this paper was submitted to the American Mathematical Society in February, 1959. See Notices Amer. Math. Soc. vol. 6 (1959) p. 281.

${ }^{2}$ In this paper the word "imbedded" will always mean "imbedded differentiably without self intersections." For immersions (with self intersections allowed) the answer to this question is negative. Examples of S. Smale show $S^{2}$ may be immersed in $R^{4}$ with a variety of normal bundles.

3 The author is grateful to Kervaire for sending him a copy of [4] before publication. 
TheOREM. Let $M^{n}$ be a compact, differentiable, orientable manifold such that the integral homology groups $H_{i}\left(M^{n}\right)$ vanish for $0<i<n$ (i.e. $M^{n}$ is a homology sphere. It need not be simply connected). Then the normal bundle to $M^{n}$ is of the same fibre homotopy type as a product bundle for any imbedding in Euclidean space of any dimension.

We will give two proofs of this theorem in $\$ 2$, both based on the Alexander duality theorem and the following simple criterion given by A. Dold [2] for a fibre bundle to be of the fibre homotopy type of a product bundle: Let $p: E \rightarrow B$ be a fibre bundle with locally compact fibre $F$ and base space $B$ a polyhedron. Then $E$ is fibre homotopy equivalent to the product bundle $B \times F$ if and only if there exists a continuous map $h: E \rightarrow F$ such that $h$ restricted to any fibre is a homotopy equivalence.

Corollary. Let $M^{n}$ be a differentiable manifold which is homeomorphic to an $n$-sphere. Then the normal bundle to $M^{n}$ is trivial for any imbedding in $(n+3)$-dimensional Euclidean space.

The corollary follows from the main theorem by applying results of A. Dold [2] on the fibre homotopy type of sphere bundles over spheres. It is proved in $\$ 3$.

By using this corollary and statements (b) and (c) above, one sees that for $n \leqq 6$, any imbedding of an $n$-sphere in Euclidean space (of any dimension) gives rise to a trivial normal bundle. It is an open question whether or not it is possible to imbed a 7 -sphere in Euclidean 11 -space with a nontrivial normal bundle.

2. Proof of the theorem. First, we will prove statement (b) of the introduction, thus disposing of the case of an orientable $n$-manifold imbedded differentiably in $(n+1)$-dimensional or $(n+2)$-dimensional Euclidean space. In the case of an $n$-manifold in $(n+1)$-space, it is obvious that the normal bundle is trivial. It is also trivial for the case of an orientable $n$-manifold imbedded in $(n+2)$-space, because of the following two facts:

(2.1) Let $M^{n}$ be a compact orientable $n$-manifold imbedded differentiably in $R^{n+k}$. Then the characteristic class of the normal bundle, $W_{k} \in H^{k}\left(M^{n}, Z\right)$, vanishes. This is an old theorem of Seifert and Whitney. For a modern proof, see Thom [6, Corollary III. 15]

4 Two fibre bundles $p_{1}: E_{1} \rightarrow B$ and $p_{2}: E_{2} \rightarrow B$ over the same base space $B$ are of the same fibre homotopy type if the:e exist fibre preserving maps $f: E_{1} \rightarrow E_{2}$ and $g: E_{2} \rightarrow E_{1}$ such that the composed maps $f g$ and $g f$ are both homotopic to the respective identity maps, with homotopies that preserve the fibres (i.e., are compatible with the equivalence relation defined by the fibration). This concept was introduced by Thom in Chapter IV of his thesis [6]. 
(2.2) The equivalence class of an $S O(2)$-bundle $^{5}$ over a given base space $B$ is completely determined by the characteristic class $W_{2}$ $\in H^{2}(B, Z)$ (this follows from the fact that the classifying space for the group $S O(2)$ is an Eilenberg-MacLane space, $K(Z, 2)$ ).

Before proceeding to the proof of the main theorem, it is convenient to state and prove a lemma.

(2.3) Lemma. Let $M^{n}$ be a compact manifold which is imbedded differentiably in an $(n+k)$-sphere, $S^{n+k}$, with $k>2$. Then the complement $S^{n+k}-M^{n}$ is simply connected.

Although this result is well known, we sketch a proof for the sake of completeness. Let $f: S^{1} \rightarrow\left(S^{n+k}-M^{n}\right)$ be a map of a circle into the complement. Using standard approximation theorems, we may as well assume that $f$ is a differentiable map. Since $S^{n+k}$ is simply connected, there exists a singular 2-cell $F: E^{2} \rightarrow S^{n+k}$ such that $F \mid S^{1}=f$, where $E^{2}$ is a disc having $S^{1}$ as boundary. Again, by the use of known approximation theorems, we may as well assume that $F$ is differentiable. Of course $F\left(E^{2}\right)$ may intersect $M^{n}$; however, by use of Theorem 1.5 of Thom [7], we see that by altering $F$ by an arbitrarily small amount in a small neighborhood of $F^{-1}\left(M^{n}\right)$, we may obtain a map $F_{1}: E^{2} \rightarrow S^{n+k}$ such that $F_{1}^{-1}\left(M^{n}\right)$ is empty. This proves that the original map $f$ is homotopic to a constant in the complement of $M^{n}$.

We will now prove the theorem stated in the introduction. Let $M^{n}$ be a compact, orientable differentiable $n$-manifold which has the same integral homology groups as an $n$-sphere, and assume that $M^{n}$ is imbedded in Euclidean $(n+k)$-space, $R^{n+k}$. Let $S^{n+k}$ denote the one point compactification of $R^{n+k}$. In view of statement (b) of the introduction, we may limit ourselves to the case $k>2$; then the complement, $S^{n+k}-M^{n}$, is simply connected.

Choose a number $\epsilon>0$ so small that the set of all points of $S^{n+k}$ whose distance from $M^{n}$ is $\leqq \epsilon$ is a tubular neighborhood $T$ of the submanifold $M^{n}$ (in the sense of Thom [7, Chapter I, §3]); i.e. so that through each point $x$ whose distance from $M^{n}$ is $\leqq \epsilon$ there is a unique geodesic normal to $M^{n}$ of length $\leqq \epsilon$. Let $E$ denote the boundary of $T$, and let $A$ denote the closure of $S^{n+k}-T$. Then obviously $A$ is a deformation retract of $S^{n+k}-M^{n}$; hence $A$ is simply connected. Similarly, $M^{n}$ is a deformation retract of $T$. Note that $A \cup T=S^{n+k}$ and $A \cap T=E$. It is also known that $E$ and $T$ are fibre bundles over $M^{n}$, with fibres a $(k-1)$-sphere and a $k$-cell respectively, and $S O(k)$ as structure group. The bundle $E$ is naturally isomorphic to the bundle

s The notation $S O(n)$ denotes the group of all $n \times n$ real orthogonal matrices of determinant +1 . 
of unit normal vectors to $M^{n}$. We will now give two slightly different proofs that $E$ has the fibre homotopy type of $M^{n} \times S^{k-1}$ by using the criterion of A. Dold.

First Proof. Let $S^{k-1}$ be a typical fibre of $E$. It is clear that the fundamental cycle on $S^{k-1}$ and the fundamental cycle on $M^{n}$ have linking number \pm 1 , depending on the orientations chosen. For, $S^{k-1}$ bounds a $k$-cell of the bundle $T$ and this $k$-cell intersects $M^{n}$ orthogonally and in a single point. From the Alexander duality theorem, it is clear that the homology groups of $S^{n+k}-M^{n}$ are isomorphic to those of a $(k-1)$-sphere. Moreover, from the version of the Alexander duality theorem as given in (say) [1, Chapter 11], it is clear that the fundamental cycle on $S^{k-1}$ is a generating cycle for the $(k-1)$ dimensional homology group of $S^{n+k}-M^{n}$. Therefore, the inclusion map $S^{k-1} \rightarrow\left(S^{n+k}-M^{n}\right)$ induces an isomorphism of homology groups in all dimensions. By Runge's theorem [1, pp. 143-146] $S^{n+k}-M^{n}$ is triangulable. Since it is simply connected, a theorem of J. H. C. Whitehead (see [3, Chapter VII, Theorem 3.8]) can be applied to conclude that the inclusion $S^{k-1} \rightarrow\left(S^{n+k}-M^{n}\right)$ is a homotopy equivalence. We may now apply the criterion of A. Dold (Corollary 2 to Theorem 1 of [2]) to the inclusion map $E \rightarrow\left(S^{n+k}-M^{n}\right)$ to conclude that $E$ has the fibre homotopy type of a product bundle.

SECond Proof. Consideration of the Mayer-Vietoris sequence of the triad $\left(S^{n+k} ; A, T\right)$ shows that the homomorphisms $j_{1}: H_{n}(E)$ $\rightarrow H_{n}(T)$ and $j_{2}: H_{k-1}(E) \rightarrow H_{k-1}(A)$ (induced by the inclusions) are isomorphisms onto in case $k-1 \neq n$, while if $k-1=n$, they are both onto, and $H_{n}(E)$ is the direct sum of the two kernels.

Since the characteristic class of the normal bundles vanishes according to (2.1), it follows that any fibre $S^{k-1}$ is totally nonhomologous to zero, i.e., the homomorphism $i_{*}: H_{k-1}\left(S^{k-1}\right) \rightarrow H_{k-1}(E)$ is an isomorphism into (see Serre [5, Chapter III]).

Combining these two facts, we see that the composition of the two homomorphisms

$$
H_{k-1}\left(S^{k-1}\right) \stackrel{i_{*}}{\rightarrow} H_{k-1}(E) \stackrel{j_{1}}{\rightarrow} H_{k-1}(A)
$$

is an isomorphism onto. This is obvious if $n \neq k-1$, while if $n=k-1$, the following slightly more elaborate argument is needed. Consider the following commutative diagram:

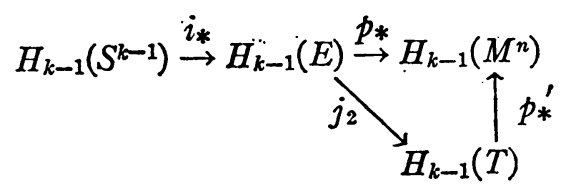


Here $p: E \rightarrow M^{n}$ and $p^{\prime}: T \rightarrow M^{n}$ are projections of the fibre bundle structures mentioned earlier. By Proposition 5, Chapter III of Serre's thesis [5], the top line of this diagram is exact. The projection $p^{\prime}$ is obviously a homotopy equivalence; hence $p_{*}^{\prime}$ is an isomorphism onto. It follows that image $i_{*}=$ kernel $j_{2}$. Since $H_{k-1}(E)$ is the direct sum of kernel $j_{1}$ and kernel $j_{2}$, it follows that $j_{1}$ maps image $i_{*}$ isomorphically on to $H_{k-1}(A)$.

In either case, we can now apply a theorem of J. H. C. Whitehead (Corollary 1 to Theorem 3 of [8]) to conclude that $j_{1} \circ i: S^{k-1} \rightarrow A$ is a homotopy equivalence. For, we have already pointed out that $A$ is simply connected, and $A$ is an absolute neighborhood retract since it is locally contractible and finite dimensional. Moreover, $H_{i}(A)=0$ except for $i=0$ and $i=k-1$ by the Alexander duality theorem.

We can now apply the criterion of A. Dold (Corollary 2 to Theorem 1 of [2]) to the inclusion map $E \rightarrow A$ to conclude that $E$ has the fibre homotopy type of a product bundle.

3 . Proof of the corollary. Let $S^{n}$ be an $n$-sphere differentiably imbedded in $R^{n+3}$; we will prove that the normal bundle to $S^{n}$ is trivial.

By the theorem just proved, the normal bundle has the same fibre homotopy type as the product bundle, $S^{n} \times S^{2}$. Therefore, the homotopy class of the characteristic map of the normal bundle belongs to the kernel of the homomorphism

$$
j_{*}: \pi_{n-1}(S O(3)) \rightarrow \pi_{n-1}\left(\mathrm{G}^{2}\right)
$$

by Theorem 4 of Dold [2] (here $\mathcal{G}^{2}$ denotes the space of all maps of $S^{2}$ onto itself of degree +1 and $j: S O(3) \rightarrow \mathcal{G}^{2}$ denotes the inclusion). Now consider the following commutative diagram of Dold (loc. cit., p. 130):

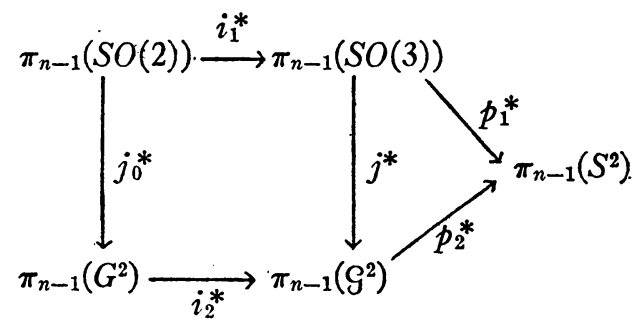

For $n>2, \pi_{n-1}(S O(2))=0$; hence $p_{1}^{*}$ is an isomorphism into by exactness. From the commutativity of the triangle on the right of this diagram, it follows that $j^{*}$ is an isomorphism into. Thus the kernel of $j^{*}$ is 0 ; hence the characteristic map of the normal bundle is null- 
homotopic. It follows that the normal bundle is trivial. In case $n \leqq 2$, the result is easily proved by other methods, or Kervaire's theorem (statement (c) in the Introduction) can be used.

\section{BIBLIOGRAPHY}

1. P. Alexandroff and H. Hopf, Topologie. Berlin, Julius Springer, 1935.

2. A. Dold, Über fasernweise Homotopieäquivalenz von Faserräumen, Math. Z. vol. 62 (1955) pp. 111-136.

3. P. J. Hilton, An introduction to homotopy theory, Cambridge, The University Press, 1953.

4. M. Kervaire, An interpretation of G. Whitehead's generalization of H. Hopf's invariant, Ann. of Math. vol. 69 (1959) pp. 345-364.

5. J.-P. Serre, Homologie singulière des espaces fibrés, Ann. of Math. vol. 54 (1951) pp. $425-505$.

6. R. Thom, Espaces fibrés en spheres et carrés de Steenrod, Ann. École Norm. Sup. vol. 69 (1952) pp. 109-182.

7. R. Thom, Quelques propriétés globales des variêtés differentiables, Comment. Math. Helv. vol. 28 (1954) pp. 17-86.

8. J. H. C. Whitehead, On the homotopy type of $A N R$ 's, Bull. Amer. Math. Soc. vol. 54 (1948) pp. 1133-1144.

Brown UNIVERSITY 